\title{
L'équation aux variations verticales d'un équilibre relatif comme source de nouvelles solutions périodiques du problème des N corps
}

\author{
Alain Chenciner ${ }^{\mathrm{a}, \mathrm{b}} \&$ Jacques Féjoz ${ }^{\mathrm{a}, \mathrm{c}}$ \\ ${ }^{\mathrm{a}}$ Astronomie et Systèmes dynamiques, IMCCE (UMR 8028 du CNRS), Observatoire de Paris \\ b Département de Mathématiques, Université D. Diderot, Paris \\ ${ }^{\mathrm{c}}$ Analyse algébrique, Institut de Mathématiques (UMR 7586 du CNRS), Université P. E M. Curie, Paris
}

\section{Résumé}

À partir d'un repère tournant qui met en résonance la fréquence d'un équilibre relatif du problème des $N$ corps avec celle d'une variation périodique infinitésimale normale au plan de l'équilibre, nous obtenons par continuation une classe remarquable de solutions périodiques. Le premier exemple en est la famille $P_{12}$, découverte par Christian Marchal, qui relie l'équilibre relatif de Lagrange au Huit.

\section{Abstract}

We start with a rotating frame which enforces a resonance between the frequency of a relative equilibrium of the $N$-body problem and that of an infinitesimal variation normal to the plane of the equilibrium. Continuation then yields a remarkable class of periodic solutions. The first example is the $P_{12}$ family, discovered by Christian Marchal, which links the relative equilibrium of Lagrange to the Eight.

\section{Abridged English version}

Provided one knows the so-called central configurations, the homographic (i.e. Kepler-like) solutions of the $N$-body problem are the simplest solutions and the only "explicit" ones. Moreover, they are always planar if not homothetic. Among them, even simpler are the relative equilibria, where each body has a circular motion with constant velocity around the center of mass. We propose to build new families of relative periodic solutions by taking advantage of the rotational invariance of these particular solutions. More precisely, calling "horizontal" the plane of motion of a relative equilibrium, we notice that the variational equation along such a solution splits into horizontal and vertical parts. Moreover, due to the attractive character of the Newtonian force, every solution of the vertical part is quasi-periodic. In general, its normal modes give rise to Lyapunov families of periodic solutions after a reduction by the $S O(2)$-symmetry which transforms the relative equilibrium into an isolated equilibrium. To these

Email addresses: chencine@imcce.fr (Alain Chenciner), fejoz@math.jussieu.fr (Jacques Féjoz). 
families correspond invariant domains in the phase space, diffeomorphic to $\mathbb{S}^{1} \times \mathbb{R}^{2}$. These domains are foliated by families of quasi-periodic solutions of the $N$-body problem which contain infinitely many genuinely periodic solutions. Each of these periodic solutions can, in turn, be continued back to the relative equilibrium solution as a family of periodic solutions in a rotating frame. We look for these by reversing the process, but now fixing the period rather than the angular momentum : appropriately choosing a rotating frame into which the infinitesimal solution defined by a particular solution of the "vertical variational equation" becomes periodic (there are infinitely many such choices), we try to continue this solution, keeping as much as possible of its discrete symmetry type, into a family of relative periodic solutions parametrized by the amount of rotation of the frame. If the family continues up to zero rotation, we get a true periodic solution. In this note, we give examples emanating from the simplest relative equilibria of $N$ equal masses, those whose configuration is the regular $N$-gon. We already recover recently discovered periodic solutions like the Eight for 3 or 5 bodies, the Hip-Hop for 4 bodies and the chain with four lobes for 5 bodies. Moreover, a vertical chain with an even number of lobes gives rise to two families which join at the chain, are symmetric with respect to one another around the vertical plane and end up at the corresponding relative equilibrium with opposite orientations. When rotated around the vertical axis, these two families form an invariant 3-dimensional lens space, which is obtained by gluing two copies of $\mathbb{S}^{1} \times \mathbb{D}^{2}$ along their boundaries in a manner depending on the number of lobes. In the case of the Eight for 3 bodies (Marchal's $P_{12}$-family) or the Hip-Hop for 4 bodies, the whole family is made of action minimizers and hence can be shown to exist. In other cases, the evidence is for the moment only numerical. The applicability of the method seems quite wide, including the cases of different masses, more complicated central configurations and higher space dimension.

1. L'équation aux variations verticales d'un équilibre relatif. Soit $x(t)=\left(\vec{r}_{1}(t), \ldots, \vec{r}_{N}(t)\right)$ une solution d'équilibre relatif de période $T_{1}$ des équations du problème newtonien des $N$ corps dans l'espace euclidien $\mathbb{R}^{3} \equiv \mathbb{C} \times \mathbb{R}:$

$$
m_{i} \ddot{\vec{r}}_{i}=\sum_{j \neq i} m_{i} m_{j} \frac{\vec{r}_{j}-\vec{r}_{i}}{\left\|\vec{r}_{j}-\vec{r}_{i}\right\|^{3}} .
$$

Dans une telle solution, la configuration $C$ formée par les corps est indépendante du temps et on qualifie de centrales les configurations admettant de tels mouvements. De plus, une telle solution est nécessairement dans un plan fixe, qu'on identifiera à $\mathbb{C} \times\{0\}$ et qu'on appellera « horizontal ». Dorénavant, on supposera donc que, pour tout $j=1,2, \ldots, N$, on a $\vec{r}_{j}(t)=\left(u_{j}(t), 0\right) \in \mathbb{C} \times\{0\}$.

L'équation aux variations le long d'une solution quelconque située dans le plan horizontal se scinde en une partie verticale et une partie horizontale. C'est une conséquence immédiate de ce qu'une variation infinitésimale des positions dans la direction verticale ne change pas au premier ordre les distances entre les corps. Si les $r_{i j}=\left\|\vec{r}_{i}-\vec{r}_{j}\right\|$ sont les distances mutuelles de notre configuration centrale $C$, qui sont des constantes du mouvement, l'équation aux variations verticales le long de l'équilibre relatif $x(t)$ s'écrit :

$$
m_{i} \ddot{\xi}_{i}=\sum_{j \neq i} \frac{m_{i} m_{j}}{r_{i j}^{3}}\left(\xi_{j}-\xi_{i}\right) .
$$

Le centre de gravité d'une solution périodique est nécessairement fixe et nous le supposerons à l'origine de $\mathbb{R}^{3}$. Nous ne considérerons donc que des variations $\left(\xi_{1}, \xi_{2}, \ldots \xi_{N}\right)$ telles que $\sum_{i=1}^{N} m_{i} \xi_{i}=0$. De l'attractivité de la force newtonienne on déduit, via la proposition 2.8 de [AC] (d'ailleurs valable en dimension d'espace quelconque), que ( $V V E$ ) possède une base de solutions périodiques, les modes propres, dont nous noterons $\omega_{1}, \ldots, \omega_{k}$ les fréquences. Nous conviendrons que $\omega_{1}=2 \pi / T_{1}$ est la fréquence de l'équilibre relatif $x(t)=(u(t), 0)$. Autrement dit, on a $u(t)=e^{i \omega_{1} t} \cdot u(0)$ et la solution « au premier ordre 》 associée à un mode propre de $(V V E)$ de vecteur propre complexe $Z \in \mathbb{C}^{N}$ s'écrit

$$
(u(t), \xi(t))=\left(e^{i \omega_{1} t} u(0), \operatorname{Re}\left(Z e^{i \omega_{j} t}\right)\right) .
$$


2. Une remarque. Soit $\Xi(t)=\left(\xi_{1}(t), \ldots, \xi_{N}(t)\right)$ une solution périodique de $(V V E)$ de fréquence $\omega_{j}=$ $2 \pi / T_{j}$. Observons-la dans un repère tournant autour de l'axe vertical à une vitesse constante choisie de façon à mettre $\Xi(t)$ et $x(t)=(u(t), 0)$ en résonance. Plus précisément, la configuration dans le repère tournant $X(t)=(U(t), 0)$ est définie par $u(t)=e^{i \varpi t} \cdot U(t)$, où $\varpi$ est choisie de façon qu'il existe des entiers $p$ et $q$ tels que $p\left(\omega_{1}-\varpi\right)=q \omega_{j}$. Cette dernière condition équivaut à ce que, dans le repère tournant, la «solution infinitésimale » $\left(\left(u_{1}(t), \xi_{1}(t)\right), \ldots,\left(u_{N}(t), \xi_{N}(t)\right)\right)$ devienne périodique de période

$$
T=\frac{2 \pi q}{\omega_{1}-\varpi}=\frac{2 \pi p}{\omega_{j}}
$$

Si elle ne correspond pas à une simple rotation du plan du mouvement, cette solution infinitésimale brise la symétrie continue de la solution d'équilibre relatif en la transformant en une symétrie discrète dans le repère tournant. En général elle engendre une famille à un paramètre (la fréquence de rotation du repère, variant dans un intervalle qui contient $\varpi$ ) de solutions périodiques dans le repère tournant, de même période $T$ et conservant une grande partie de cette symétrie. Pour peu que l'intervalle de variation du paramètre contienne 0 (éventuellement après régularisation des collisions doubles), on obtiendra une solution périodique avec ces symétries dans le repère initial.

Pour comprendre l'origine de cette méthode, effectuons une réduction de la symétrie de rotation autour de l'axe vertical, qui transforme l'équilibre relatif $x(t)$ en un équilibre isolé. Aux solutions périodiques de (VVE) correspondent en général des familles de Lyapunov de solutions périodiques du système réduit (voir [CFM2] pour une démonstration dans le cas de l'équilibre de Lagrange de 3 masses égales, et [MS] dans le cas du polygone régulier avec une masse centrale). Une telle famille est la projection d'un domaine de l'espace des phases difféomorphe à $\mathbb{S}^{1} \times \mathbb{R}^{2}$, feuilleté par une famille de solutions quasipériodiques du problème des $N$ corps. Parmi celles-ci, les solutions périodiques sont en général denses. De chacune de ces solutions périodiques on peut revenir à l'équilibre relatif à travers une famille de solutions périodiques dans un repère tournant. La méthode proposée revient à renverser le processus en fixant la période plutôt que le moment cinétique et à utiliser les symétries pour choisir la famille de solutions.

Nous illustrons cette remarque par des exemples issus de l'équilibre relatif de la configuration centrale la plus simple, le $N$-gone régulier pour $N$ masses égales ([CF]). Dans le cas des chaînes verticales à un nombre pair $2 k$ de lobes, il existe deux familles symétriques par rapport au plan vertical, distinguées par l'orientation de l'équilibre relatif de départ. Si l'on adjoint aux solutions de ces deux familles celles obtenues par rotation autour de l'axe vertical, on obtient dans l'espace des phases un espace lenticulaire $L(4 k, 1)$ invariant, recollement sur leur bord des tores solides formés par les deux familles.

Exemple 1 (3 corps) : le Huit. Comme l'a très rapidement compris Christian Marchal, le Huit ([CM]) peut être obtenu par le procédé indiqué en partant de l'équilibre relatif de Lagrange de trois masses égales. C'est la «famille $P_{12} »([\mathrm{M}],[\mathrm{C} 1],[\mathrm{C} 3],[\mathrm{CFM} 1])$ dans laquelle le cercle parcouru deux fois s'ouvre comme une huître jusqu'à s'aplatir complètement, puis se referme symétriquement. Sur la figure, $\varpi / \omega_{1}=-1, \simeq-1+10^{-2}, 0, \simeq 1-10^{-2}, 1$ (les échelles horizontale et verticale sont distinctes et variables) :
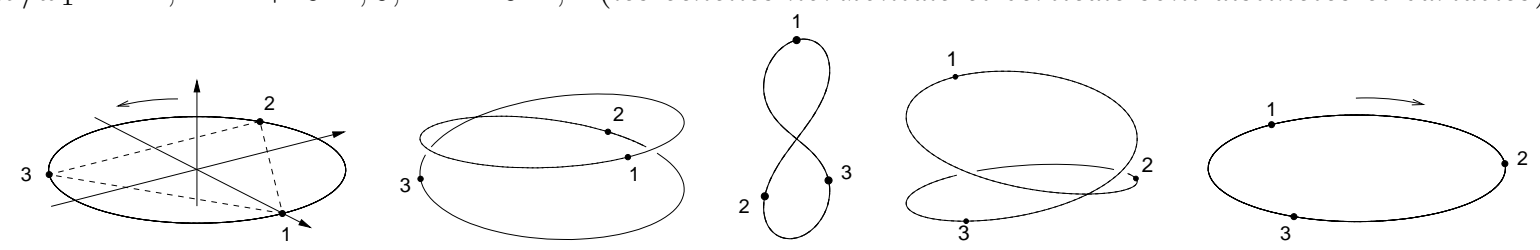

Il y a ici une seule fréquence fondamentale, égale à $\omega_{1}$, pour l'équation aux variations. On a

$$
\omega_{j}=\omega_{1}, \varpi=-\omega_{1}, p=1, q=2 .
$$


Lorsque la fréquence de rotation du repère varie de $-\omega_{1}$ à 0 , les éléments de la famille minimisent l'action. Pour des valeurs inférieures à $-\omega_{1}$ de cette fréquence, c'est la solution d'équilibre relatif du triangle qui minimise l'action sous les contraintes données de symétrie ([M], [C1], [C3]).

Exemple 2 (4 corps) : le Hip-Hop. Cette solution [CV] du problème des 4 corps de masses égales peut être obtenue à partir de la solution d'équilibre relatif du carré $([\mathrm{CF}])$. Il y a deux fréquences fondamentales $\omega_{1}$ et $\omega_{2}=\frac{2 \omega_{1}}{\sqrt{2+1 / \sqrt{2}}}$ pour l'équation aux variations. On prend ici :

$$
\omega_{j}=\omega_{2}, \varpi=\omega_{1}-\omega_{2}, p=1, q=1 .
$$

Comme ci-dessus, lorsque la fréquence de rotation du repère varie de $\omega_{1}-\omega_{2}$ à 0 , les éléments de la famille minimisent l'action. Pour des valeurs inférieures à $-\omega_{1}$ de cette fréquence, c'est la solution d'équilibre relatif du carré qui minimise l'action sous les contraintes données de symétrie. Sur la figure, $\varpi / \omega_{1}=$ $1-\omega_{2} / \omega_{1}, \simeq 1-\omega_{2} / \omega_{1}+10^{-3}, 0:$
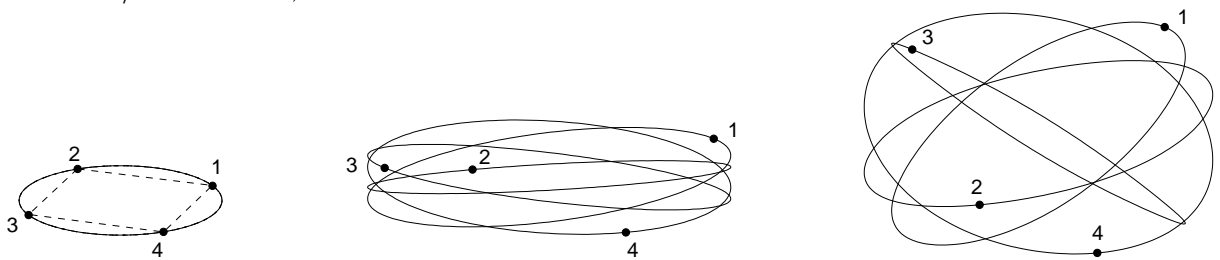

Par contre, le choix de $\omega_{j}=\omega_{1}, \varpi=-2 \omega_{1}, p=1$ et $q=3$ fournit numériquement un dépliement en 3 lobes du cercle parcouru 3 fois, qui aboutit, pour $\varpi \simeq-0.72 \varpi_{1}$, à une solution plane horizontale passant très près d'une collision. La continuation naturelle de cette famille est sa symétrisée par rapport au plan horizontal. Quant à la solution plane, elle se continue jusqu'à la solution de Gerver, ce qui précise la figure 2.6 de $[\mathrm{B}]$ (merci à S. Terracini d'avoir attiré notre attention sur cette figure). Sur la figure, $\varpi / \omega_{1}=-2, \simeq-2+10^{-2}, \simeq-0.9, \simeq-0.72$ :
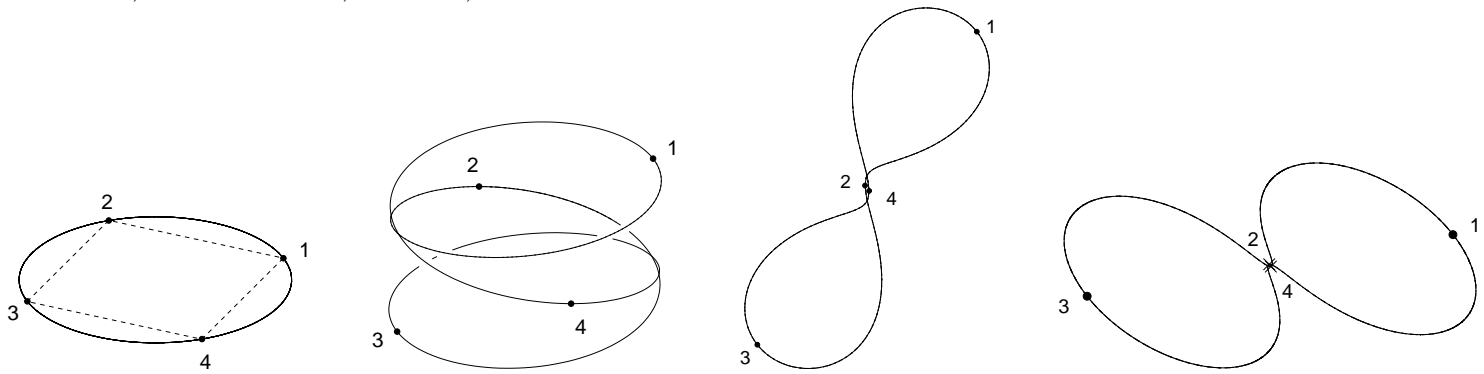

La différence avec le Huit est que, pour un nombre pair de corps, les symétries de la chaîne à trois lobes (terminologie de [CGMS]) n'impliquent pas que le moment cinétique soit nul; or, ce dernier doit être porté par l'axe de rotation, donc vertical. Ainsi, une solution plane de la famille dont le moment cinétique n'est pas nul est nécessairement horizontale.

Exemple 3 (5 corps) : la chaîne à 4 lobes et le Huit. Cette solution, trouvée numériquement par Simó ([S], figure 1), peut être obtenue à partir de l'équilibre relatif du pentagone régulier (5 masses égales). Ici, c'est le cercle parcouru 4 fois qui se déploie en 4 lobes finissant par s'aplatir ([CF]). Comme pour le Huit, la solution obtenue est automatiquement de moment cinétique nul car dans un plan contenant l'axe de rotation. L'équation aux variations possède deux fréquences propres $\omega_{1}$ et $\omega_{2}$, et c'est d'une solution de fréquence $\omega_{1}$ que l'on part : $\omega_{j}=\omega_{1}, \varpi=-3 \omega_{1}, p=1, q=4$. 
Sur la figure, $\varpi / \omega_{1}=-3, \simeq-3+2 \times 10^{-3}, \simeq-1.6,0$ :
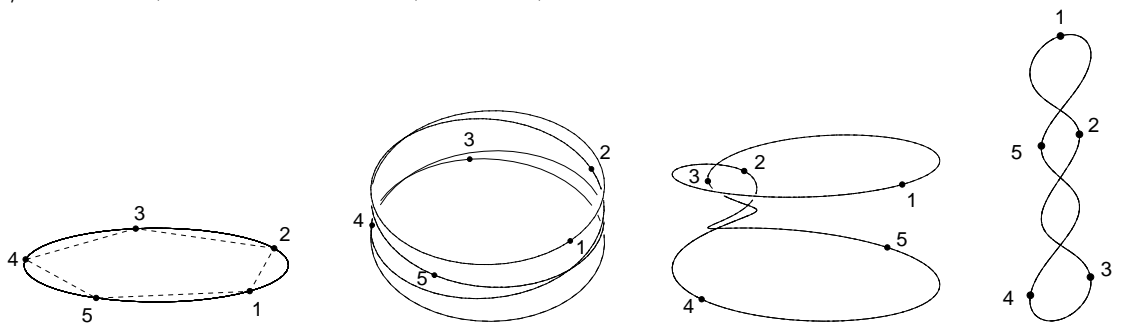

Ici, la minimisation de l'action sous les contraintes de symétrie données ne conduit pas à la chaîne à 4 lobes mais plutôt à la chorégraphie du Huit à 5 corps (voir ci-dessous). Une preuve mathématique de l'existence de cette famille n'existe donc pas pour l'instant.

Pour obtenir le Huit avec 5 corps, il faut partir d'une solution de l'équation aux variations de fréquence $\omega_{2}$ en choisissant $\varpi=-\left(2 \omega_{2}-\omega_{1}\right)$ et donc $p=1, q=2$. Sur la figure, $\varpi / \omega_{1} \simeq-1.7,-1.2,-0.8,0$ :
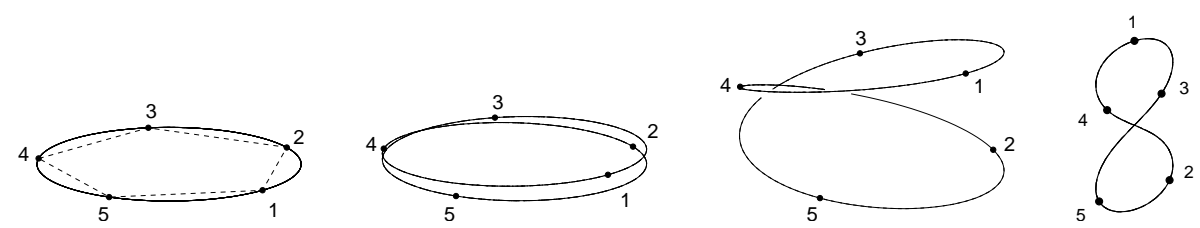

Mais il y a plus : nous n'avons considéré jusqu'ici que la plus symétrique des configurations centrales, le polygone régulier pour des masses égales, à laquelle sont naturellement reliés les chorégraphies et les HipHops symétriques. Et même dans ce cas, nous avons choisi les rotations du repère qui se présentent le plus immédiatement. Dans le cas du Hip-Hop de 4 corps, nous aurions pu par exemple, ou bien transformer la solution infinitésimale en une chorégraphie par une rotation supplémentaire du repère (d'un demi-tour par période pour la plus simple) et obtenir sans doute les chorégraphies spatiales de [TV], ou bien considérer $N \geq 4$ masses quelconques et, partant d'une configuration centrale qui minimise le potentiel à inertie constante, obtenir les Hip-Hops généralisés de [C2] (voir également [C3]).

Revenant au cas de masses égales, l'analyse de l'équation aux variations verticales fait conjecturer qu'à partir de l'équilibre relatif du $N$-gone régulier on obtiendra, pour $N$ impair, les chaînes verticales à un nombre pair $2 p \leq N-1$ de lobes dont les propriétés de symétrie impliquent un moment cinétique nul. Pour les chaînes à un nombre impair de lobes, le scénario le plus simple passe par une solution plane horizontale en repère tournant, dont la continuation dans le plan horizontal conduit à la chaîne en repère fixe. Plus généralement, la classification dépendra des propriétés de divisibilité de $N$.

Remerciements Ayant reçu une première version de cette note, Michel Hénon a attiré notre attention sur les deux articles [H1,H2]. Il y étudie les bifurcations de solutions périodiques tridimensionnelles du problème restreint circulaire des 3 corps à partir de ce qu'il appelle les solutions périodiques planes « critiques », c'est-à-dire marginalement stables (un équilibre relatif est automatiquement critique). Bien que dans un autre contexte, la figure 17 de [H1] rappelle les courbes 2 et 4 de notre première figure. Nous sommes également redevables à Michel Hénon de nous avoir indiqué l'existence des nombreux travaux que $[\mathrm{H} 1, \mathrm{H} 2]$ ont suscités : les auteurs qui ont suivi explorent, souvent numériquement, la bifurcation de solutions spatiales du problème des trois corps à partir de solutions planes critiques. Mais, à notre connaissance, aucun n'a exploité systématiquement comme nous le faisons les symétries des solutions issues des équilibres relatifs. 


\section{Références}

[AC] A. Albouy \& A. Chenciner, Le problème des $n$ corps et les distances mutuelles, Inventiones Mathematicae, 131, 151-186 (1998)

[B] V. Barutello, Thèse, U. Milano Bococca, chapitre 2 (2004)

[C1] A. Chenciner, Some facts and more questions about the "Eight", Proceedings de la conférence "Nonlinear Functional Analysis", Taiyuan (Shanxi) août 2002, p. 77-88 du livre "Topological methods, Variational methods and their applications", H. Brezis, K.C. Chang, S.J. Li, P. Rabinowitz éditeurs, World Scientific (2003)

[C2] A. Chenciner, Simple non-planar periodic solutions of the $n$-body problem, proceedings du colloque NDDS de Kyoto (août 2002)

[C3] A. Chenciner, Action minimizing solutions of the $n$-body problem : from homology to symmetry, Proceedings du Congrès international des mathématiciens (ICM), Pékin, août 2002, vol. III, 279-294

[CFM1] A. Chenciner, J. Féjoz \& R. Montgomery, Rotating Eights I : the three $\Gamma_{i}$ families, soumis à Non Linearity.

[CFM2] A. Chenciner, J. Féjoz \& R. Montgomery, Rotating Eights II, en préparation

[CF] A. Chenciner \& J. Féjoz, The regular polygon unchained, en préparation

[CGMS] A. Chenciner, J.Gerver, R.Montgomery \& C.Simó, Simple Choreographic Motions of $N$ bodies : A Preliminary Study, in Geometry, Mechanics, and Dynamics, volume dédié à J. Marsden, pp. 287-308, Springer (2002)

$[\mathrm{CM}]$ A.Chenciner \& R.Montgomery, A remarkable periodic solution of the three-body problem in the case of equal masses, Annals of Mathematics 152, 881-901 (2000)

[CV] A. Chenciner \& A.Venturelli, Minima de l'intégrale d'action du Problème newtonien de 4 corps de masses égales dans $\mathbb{R}^{3}$ : orbites "hip-hop", Celestial Mechanics 77, 139-152 (2000)

[H1] M. Hénon, Vertical stability of periodic orbits in the restricted problem. 1. Equal masses, Astron. Astrophys. 28 $415-426(1973)$

[H2] M. Hénon, Vertical stability of periodic orbits in the restricted problem. 2. Hill's case, Astron. Astrophys. 30 $317-321(1974)$

[M] C. Marchal, The family $P_{12}$ of the three-body problem - the simplest family of periodic orbits, with twelve symmetries per period, Celestial Mechanics 78, 279-298 (2000)

[MS] K. Meyer \& D. Schmidt, Libration of central configurations and braided Saturn rings, Celestial Mechanics 55, 289-303 (1993)

[S] C. Simó, New families of Solutions in N-Body Problems, Proceedings of the Third European Congress of Mathematics, C. Casacuberta et al. eds., Progress in Mathematics 201, 101-115 (2001)

[TV] S. Terracini \& A. Venturelli, Hip-Hop type solutions with 2N equal masses, preprint (2004) 